\title{
DERIVATIVES OF HARDY FUNCTIONS
}

\author{
BOO RIM CHOE
}

(Communicated by Clifford J. Earle, Jr.)

\begin{abstract}
Let $B$ be the open unit ball of $C^{n}$, and set $S=\partial B$. It is shown that if $\varphi \in L^{p}(S), \varphi>0$, is a lower semicontinuous function on $S$ and $1 / q>1+1 / p$, then, for a given $\varepsilon>0$, there exists a function $f \in H^{p}(B)$ with $f(0)=0$ such that $\left|f^{*}\right|=\varphi$ almost everywhere on $S$ and $\int_{B}|\nabla f|^{q} d V<\varepsilon$ where $V$ denotes the normalized volume measure on $B$.
\end{abstract}

\section{INTRODUCTION}

Let $B$ be the open unit ball of $C^{n}$, and set $S=\partial B$. The rotation-invariant probability measure on $S$ will be denoted by $\sigma$. The Hardy space $H^{p}(B)$, $0<p<\infty$, is then the space of holomorphic functions on $B$ for which

$$
\sup _{0<r<1} \int_{S}|f(r \zeta)|^{p} d \sigma(\zeta)<\infty .
$$

For $p=\infty, H^{\infty}(B)$ denotes the space of bounded holomorphic functions on $B$. As is well known, to each $H^{p}$-function $f$ corresponds its boundary value $f^{*}$ defined almost everywhere on $S$ by $f^{*}(\zeta)=\lim _{r / 1} f(r \zeta)$; the term "almost every" will always refer to the measure $\sigma$. A remarkable fact about these boundary values of $H^{p}$-functions is that they can be prescribed as in the following.

Theorem A (Alexandrov [1]). Suppose $\varphi>0$ is a lower semicontinuous function on $S$ and $\varphi \in L^{p}(\sigma)$ for some $0<p \leq \infty$. Then there exists a function $f \in H^{p}(B)$ with $f(0)=0$ such that $\left|f^{*}\right|=\varphi$ almost everywhere on $S$.

At least for $n>1$, it seems to be the price we pay for prescribing boundary values of $H^{p}$-functions as above that their derivatives may get out of control in general. Roughly speaking, when $n>1$, a theorem of Rudin [5] shows that if $f \in H^{\infty}(B)$ is nonconstant and $\left|f^{*}\right|=1$ on some open subset $\Gamma$ of $S$, then $f$ must be extremely oscillatory near a $G_{\delta}$ dense subset $\Omega$ of $\Gamma$, so that its derivatives may get huge near $\Omega$. One such example (when $n>1$ ) is the fact [2] that if $f$ is inner (i.e., $f \in H^{\infty}(B)$ and $\left|f^{*}\right|=1$ almost everywhere on

Received by the editors October 23, 1989.

1980 Mathematics Subject Classification (1985 Revision). Primary 32A35.

Key words and phrases. Derivatives, $H^{p}$-functions. 
$S)$ and if $\int_{B}|\nabla f|^{2} d V<\infty$, then $f$ must be constant. Here and elsewhere, $\nabla f=\left(\partial f / \partial z_{1}, \ldots, \partial f / \partial z_{n}\right)$ and $V$ denotes the normalized volume measure on $B$. In some special cases, however, one may have those derivatives under very good control. For example, one can choose $f$ in Theorem $\mathrm{A}$ so that $\int_{B}|\nabla f|^{q} d V$ is arbitrarily small whenever $1 / q>1+n / p$. This follows from Theorem A itself.

Proof (sketch). Let $f$ be as in Theorem A, fix an inner function $u$ on $B$ such that $u(0)=0$, and define $f_{m}=u^{m} f$ for $m=1,2, \ldots$. Then Theorem A holds with $f_{m}$ in place of $f$. Note that $f_{m}$ converges to 0 uniformly on compact subsets of $B$ and therefore so does $\left|\nabla f_{m}\right|$. Since

$$
\left|f_{m}(z)\right| \leq C_{n, p}\|\varphi\|_{L^{p}(\sigma)}\left(\frac{1}{1-|z|}\right)^{n / p} \quad(z \in B),
$$

we have by the Cauchy estimates

$$
\left|\nabla f_{m}(z)\right| \leq C_{n, p}\|\varphi\|_{L^{p}(\sigma)}\left(\frac{1}{1-|z|}\right)^{1+n / p} \quad(z \in B) .
$$

It follows that the sequence $\left|\nabla f_{m}\right|^{q}$ is dominated by an integrable function on $B$ whenever $1 / q>1+n / p$, and thus $\int_{B}\left|\nabla f_{m}\right|^{q} d V$ converges to 0 as $m \rightarrow \infty$.

In the present paper we obtain a better upper bound for $q$ in the above observation, which is the main result of the paper.

Main theorem. Let $\varphi>0$ be a lower semicontinuous function on $S$. Suppose $\varphi \in L^{p}(\sigma)$ for some $0<p \leq \infty$ and $1 / q>1+1 / p$. Then, for a given $\varepsilon>0$, there exists a function $f \in H^{p}(B)$ with $f(0)=0$ such that $\left|f^{*}\right|=\varphi$ almost everywhere on $S$ and $\int_{B}|\nabla f|^{q} d V<\varepsilon$.

The author does not know whether the upper bound $(1+1 / p)^{-1}$ for $q$ in the above theorem is sharp in general. There is, however, a reason why the upper bound cannot be bigger than 1 , as the following remark shows.

Remark. Suppose $f \in H^{p}(B)$ and $f(0)=0$. Then

$$
\left|f^{*}(\zeta)\right| \leq \int_{0}^{1}\left|\frac{\partial}{\partial t} f(t \zeta)\right| d t \leq \int_{0}^{1}|\nabla f(t \zeta)| d t
$$

for almost every $\zeta \in S$. Hence, for $q \geq 1$, we have

$$
\int_{S}\left|f^{*}\right|^{q} d \sigma \leq \int_{S} \int_{0}^{1}|\nabla f(t \zeta)|^{q} d t d \sigma(\zeta) \leq C_{n, q} \int_{B}|\nabla f|^{q} d V .
$$

It follows that if the conclusion of the main theorem holds for some $q$, then $q<1$. Note that the upper bound for $q$ in the main theorm is sharp for $p=\infty$.

\section{Proof of MAIN THeOREM}

We need prove the main theorem only for higher-dimensional cases. From now on, $n>1$ will therefore be fixed. The main idea is to modify Rudin's 
proof [5] of Theorem A so that the derivatives in question are all under control at the same time. Before going further, let us introduce some notation first. There is a nonisotropic metric $d$ on $S$ defined by

$$
d(\zeta, \eta)=|1-<\zeta, \eta>|^{1 / 2} \quad(\zeta, \eta \in S) .
$$

Since $\sigma$ is rotation-invariant, the volume $\sigma\left(Q_{\delta}(\eta)\right)$ of the corresponding balls

$$
Q_{\delta}(\eta)=\{\zeta \in S: d(\zeta, \eta)<\delta\} \quad(0<\delta<\sqrt{2})
$$

is independent of $\eta$ and thus will be denoted by $A(\delta)$. The notation $A(B)$ will denote the class of functions holomorphic on $B$ and continuous on $\bar{B}$. Finally, $\chi_{E}$ will denote the characteristic function of $E \subset S$.

Lemma 1. Let $0<p<1$. Then there are constants $\alpha=\alpha(n, p)<1$ and $\beta=\beta(n, p)>10$ with the following property: If $Q=Q_{\delta}(\eta), 0<\delta<1$, and

$$
h(z)=\frac{i\langle z, \eta\rangle}{\left[2+\beta \delta^{-2}(1-\langle z, \eta\rangle)\right]^{4 n / p}} \quad(z \in \bar{B}),
$$

then

$$
\begin{gathered}
\int_{S}|h|^{1 / 2} d \sigma<A(\delta), \\
\int_{S}\left|\chi_{Q}-\operatorname{Re} h\right|^{1 / 2} d \sigma<\alpha \cdot A(\delta), \\
\int_{B}|\nabla h|^{p} d V<\delta^{2 n+2-2 p} .
\end{gathered}
$$

Proof. For $t \geq 1$ and $z \in \bar{B}$, define

$$
h_{t}(z)=\frac{i<z, \eta>}{[2+t(1-<z, \eta>)]^{4 n / p}} .
$$

Then

$$
\left|\nabla h_{t}(z)\right|^{p} \leq \frac{1}{|2+t(1-<z, \eta>)|^{4 n}}+\frac{8 n t^{p}}{|2+t(1-<z, \eta>)|^{4 n+p}} .
$$

By [3, Proposition 17] we have the following integral formula:

$$
\int_{B} \frac{d V(z)}{|1-<z, w>|^{l}}=\left(\frac{1}{1-|w|^{2}}\right)^{l-n-1} \int_{B} \frac{d V(z)}{|1-<z, w>|^{2 n+2-l}}
$$

for $l$ real and $w \in B$. From this it is easily verified that

$$
\int_{B} \frac{d V(z)}{|2+t(1-<z, \eta>)|^{l}}<\left(\frac{1}{2 t}\right)^{n+1}
$$

holds for every $l>2 n+2$. Thus, by (4),

$$
\int_{B}\left|\nabla h_{t}\right|^{p} d V<\frac{10 n}{2^{n+1}}\left(\frac{1}{t}\right)^{n+1-p}
$$


which in turn implies (3) whenever $\beta>10$. We need find such $\beta$ for which (1) and (2) also hold.

Assume $t$ is sufficiently large so that $t \delta^{2} \geq 2$. Then the set $E_{t}=\{\zeta \in S$ : $|1-t(1-<\zeta, \eta>)|<1\}$ is contained in $Q$, and hence

$$
\int_{Q}\left|\operatorname{Re} h_{t}\right|^{2} d \sigma \geq \int_{E_{t}}\left|\operatorname{Re} h_{t}(\zeta)\right|^{2} d \sigma(\zeta) .
$$

On the right side of the above, make successive changes of variables, first $\lambda=$ $\langle\zeta, \eta\rangle$ and then $w=t(1-\lambda)$, to obtain

$$
\frac{n-1}{\pi t^{n}} \int_{D}\left|\operatorname{Im} \frac{1-t^{-1} w}{(2+w)^{4 n / p}}\right|^{2}\left(2 \operatorname{Re} w-\frac{|w|^{2}}{t}\right)^{n-2} d m(w)
$$

where $D=\{w \in \mathrm{C}:|1-w|<1\}$ and $m$ denotes the area measure on $\mathrm{C}$. From this we easily obtain a positive constant $c=c(n, p)<1$ such that

$$
\frac{1}{8} \int_{Q}\left|\operatorname{Re} h_{t}\right|^{2} d \sigma \geq\left(\frac{c}{t}\right)^{n}
$$

whenever $t \delta^{2} \geq 2$.

We now define $\beta=10 / c$. Then $\beta>10$ and [5, §3.7] shows that (1) and (2) hold with $\alpha=1-\left(c^{2} / 20\right)^{n}+\left(c^{2} / 25\right)^{n}$. This completes the proof.

Lemma 2. Let $0<p<1$. Then there is a constant $\gamma=\gamma(n, p)<1$ with the following property: If $Q=Q_{r}(\eta), 0<r<1$, and $\tau_{i}>0, i=1,2$, then there exists a function $f \in A(B)$ with $f(0)=0$ such that

$$
\begin{gathered}
|f|<1 \quad \text { on } Q, \\
|f|<\tau_{1} \quad \text { on } S \backslash Q, \\
\int_{S}|f|^{1 / 2} d \sigma<A(r), \\
\int_{S}\left|\chi_{Q}-\operatorname{Re} f\right|^{1 / 2} d \sigma<\gamma \cdot A(r), \\
\int_{B}|\nabla f|^{p} d V<\tau_{2} .
\end{gathered}
$$

Proof. Put $\tau=\min \left\{1 / 2, \tau_{1}, \tau_{2}^{1 / 2(1-p)}\right\}$ and let $\delta=\tau r$. Pick a maximal collection $\left\{\zeta_{1}, \ldots, \zeta_{N}\right\}$ of points in $Q_{r / 2}(\eta)$ subject to the condition $d\left(\zeta_{i}, \zeta_{j}\right) \geq 2 \delta$ for $i \neq j$. Then the $d$-balls $Q_{j}=Q_{\delta}\left(\zeta_{j}\right)$ are pairwise disjoint and contained in $Q$. Thus, by [4, Proposition 5.1.4]

$$
N \leq \frac{A(r)}{A(\delta)} \leq\left(\frac{r}{\delta}\right)^{2 n}=\tau^{-2 n} .
$$

Associate $h_{j}$ to $Q_{j}$ as in Lemma 1 and define

$$
f=h_{1}+\cdots+h_{N} \text {. }
$$


Obviously $f \in A(B), f(0)=0$, and $[5, \S 3.8]$ shows that $f$ satisfies $(5) \sim(8)$ with $\gamma=\left[1-(1-\alpha) 16^{-n}\right]$, where $\alpha$ is the same constant as in Lemma 1 .

Note that $|\nabla f|^{p} \leq\left|\nabla h_{1}\right|^{p}+\cdots+\left|\nabla h_{N}\right|^{p}$. Therefore, by (10) and the choice of $\tau$, we have

$$
\int_{B}|\nabla f|^{p} d V \leq N \delta^{2 n+2-2 p}<\tau^{2-2 p} \leq \tau_{2}
$$

This proves (9). The proof is complete.

In the following lemma, $P[\psi]$ denotes the Poisson integral of $\psi \in L^{1}(\sigma)$. That is,

$$
P[\psi](z)=\int_{S} \frac{1-|z|^{2}}{|z-\zeta|^{2 n}} \psi(\zeta) d \sigma(\zeta) \quad(z \in B) .
$$

Lemma 3. Suppose that $\varphi>0$ is a lower semicontinuous function on $S$ and $\varphi \in L^{1}(\sigma)$. Then, for given $0<p<1$ and $\varepsilon>0$, there exists a function $F \in H^{1 / 2}(B)$. with $F(0)=0$ such that

(a) $\operatorname{Re} F^{*}=\varphi$ almost everywhere on $S$

(b) $\operatorname{Re} F \leq P[\varphi]$ on $B$, and

(c) $\int_{B}|\nabla F|^{p} d V<\varepsilon$.

Note. In the proof of the main theorem the fact that $F(0)=0$ will not be used. However, it implies that $F$ is nonconstant as far as the lemma itself is concerned.

Proof. Let $\gamma<1$ be the same constant as in Lemma 2, and assume $\int_{S} \varphi^{1 / 2} d \sigma<$ 1 without loss of generality. We will choose by induction a sequence of functions $F_{0}, F_{1}, \ldots$ such that for $N=0,1, \ldots$,

$$
\begin{array}{ll}
\left(11_{N}\right) & F_{N} \in A(B) \text { and } F_{N}(0)=0, \\
\left(12_{N}\right) & \varphi-\operatorname{Re}\left(F_{0}+\cdots+F_{N}\right)>0 \text { on } S, \\
\left(13_{N}\right) & \int_{S}\left[\varphi-\operatorname{Re}\left(F_{0}+\cdots+F_{N}\right)\right]^{1 / 2} d \sigma<\left(\frac{1+\gamma}{2}\right)^{N}, \\
\left(14_{N}\right) \quad & \int_{S}\left|F_{N}\right|^{1 / 2} d \sigma<\left(\frac{1+\gamma}{2}\right)^{N-1}, \\
\left(15_{N}\right) \quad & \int_{B}\left|\nabla F_{N}\right|^{p} d V<\frac{\varepsilon}{2^{N+1}} .
\end{array}
$$

To begin the induction, put $F_{0} \equiv 0 ;\left(11_{0}\right) \sim\left(15_{0}\right)$ are trivial. Suppose now that $F_{0}, \ldots, F_{N}$ have been chosen so that $\left(11_{N}\right) \sim\left(15_{N}\right)$ hold. Put $\varphi_{N}=\varphi-\operatorname{Re}\left(F_{0}+\cdots+F_{N}\right)$. Then $\varphi_{N}$ is a positive lower semicontinuous function on $S$ and thus can be approximated by an increasing sequence of positive continuous functions on $S$. Therefore the proof of [5, Lemma 3.4] shows that there are positive numbers $c_{1}, \ldots, c_{j}$, and a disjoint collection 
$\left\{Q_{1}, \ldots, Q_{J}\right\}=\left\{Q_{r_{1}}\left(\eta_{1}\right), \ldots, Q_{r_{J}}\left(\eta_{J}\right)\right\}, 0<r_{j}<1$, of $d$-balls such that

$$
\int_{S}\left(\varphi_{N}-\sum_{j=1}^{J} c_{j} \chi_{Q_{j}}\right)^{1 / 2}<\frac{1-\gamma}{2} \int_{S} \varphi_{N}^{1 / 2} d \sigma
$$

and

$$
\varphi_{N}-\sum_{j=1}^{J} c_{j} \chi_{Q_{j}}>\tau \text { on } S
$$

for some positive number $\tau$. Associate $f_{j}$ to $Q_{j}$ as in Lemma 2, with $\tau_{1}=$ $\tau / 2 J c_{j}$ and $\tau_{2}=\varepsilon / 2^{N+2} J c_{j}^{p}$ for $j=1, \ldots, J$ and define

$$
F_{N+1}=c_{1} f_{1}+\cdots+c_{J} f_{J} .
$$

Then $\left(11_{N+1}\right)$ is clear, and $[5, \S 3.9]$ shows that $\left(12_{N+1}\right) \sim\left(14_{N+1}\right)$ are satisfied. Finally,

$$
\int_{B}\left|\nabla F_{N+1}\right|^{p} d V \leq \sum_{j=1}^{J} c_{j}^{p} \int_{B}\left|\nabla f_{j}\right|^{p} d V<\frac{\varepsilon}{2^{N+2}} .
$$

This proves $\left(15_{N+1}\right)$, and the construction can therefore proceed. By $\left(14_{N}\right)$

$$
\sum_{N=0}^{\infty} \int_{S}\left|F_{N}\right|^{1 / 2} d \sigma<\infty
$$

so that $F=\sum_{N=0}^{\infty} F_{N} \in H^{1 / 2}(B)$ and $F(0)=0$, since the same is true of each $F_{N}$. Next, (a) and (c) follow from $\left(13_{N}\right)$ and $\left(15_{N}\right)$, respectively. Finally, (b) follows from $\left(12_{N}\right)$ by the harmonicity of each $F_{0}+\cdots+F_{N}$. The proof is complete.

Proof of main theorem. We may assume $0<p<\infty$. Also, without loss of generality, assume $\varphi>1$ so that $\log \varphi>0$. Note that $\log \varphi \in L^{1}(\sigma)$ by Jensen's inequality and that $1 / r=1 / q-1 / p>1$ by assumption. Thus, by Lemma 3, there is a function $F \in H^{1 / 2}(B)$ such that

$$
\begin{gathered}
\operatorname{Re} F^{*}=\log \varphi \quad \text { almost everywhere on } S, \\
\operatorname{Re} F \leq P[\log \varphi] \text { on } B,
\end{gathered}
$$

and

$$
\left(\int_{B}|\nabla F|^{r} d V\right)^{1 / r}\left(\int_{S} \varphi^{p} d \sigma\right)^{1 / p}<\left(\frac{\varepsilon}{2}\right)^{1 / q}
$$

Choose an inner function $u$ on $B$ with $u(0)=0$ such that

$$
\left(\int_{B}|\nabla u|^{r} d V\right)^{1 / r}\left(\int_{S} \varphi^{p} d \sigma\right)^{1 / p}<\left(\frac{\varepsilon}{2}\right)^{1 / q}
$$


This can be done because $r<1$ (see the Introduction). We now define $f=$ $u \exp F$. Clearly $f(0)=0, f \in H^{p}(B)$ by (17), and $\left|f^{*}\right|=\varphi$ almost everywhere on $S$ by (16). Note that $\left(P\left[\varphi^{q}\right]\right)^{p / q} \leq P\left[\varphi^{p}\right]$ by Jensen's inequality. Thus, by Hölder's inequality and (18), we obtain

$$
\begin{aligned}
\int_{B}|\nabla F|^{q} P\left[\varphi^{q}\right] d V & \leq\left(\int_{B}|\nabla F|^{r} d V\right)^{q / r}\left(\int_{B} P\left[\varphi^{p}\right] d V\right)^{q / p} \\
& =\left(\int_{B}|\nabla F|^{r} d V\right)^{q / r}\left(\int_{S} \varphi^{p} d \sigma\right)^{q / p}<\frac{\varepsilon}{2} .
\end{aligned}
$$

Similarly, by (19),

$$
\int_{B}|\nabla u|^{q} P\left[\varphi^{q}\right] d V<\frac{\varepsilon}{2}
$$

It follows that

$$
\int_{B}|\nabla f|^{q} d V<\varepsilon
$$

because $|\nabla f|^{q} \leq\left(|\nabla u|^{q}+|\nabla F|^{q}\right) P\left[\varphi^{q}\right]$. The proof is complete.

Remark. Let $N_{*}(B)$ denote the Smirnov class consisting of holomorphic functions on $B$ for which

$$
\sup _{0<r<1} \int_{S} \log ^{+}\left|f_{r}\right| d \sigma<\infty
$$

and $\left\{\log ^{+}\left|f_{r}\right|\right\}$ is uniformly integrable (with respect to the measure $\sigma$ ), where $f_{r}(\zeta)=f(r \zeta)$ for $\zeta \in S$. Then a trivial modification of the above proof of the Main Theorem shows the following:

Theorem 4. Let $\varphi>0$ be a lower semicontinuous function on $S$ such that $\log \varphi \in L^{1}(\sigma)$. Then, for a given $\varepsilon>0$, there exists a function $f \in N_{*}(B)$ with $f(0)=0$ such that $\left|f^{*}\right|=\varphi$ almost everywhere on $S$ and $\exp \int_{B} \log |\nabla f| d V<$ $\varepsilon$.

\section{REFERENCES}

1. A. B. Alexandrov, Existence of inner functions in the unit ball, Mat. Sb. 118 (1982), 147-163.

2. E. Bedford and A. B. Taylor, Two applications of a nonlinear integral formula to analytic functions, Indiana Univ. Math. J. 29 (1980), 463-465.

3. B. R. Choe, Projections, the weighted Bergman spaces, and the Bloch space, Proc. Amer. Math. Soc. 108 (1990), 127-136.

4. W. Rudin, Function theory in the unit ball of $\mathrm{C}^{n}$, Springer, New York, 1980.

5. _ Inner functions in the unit ball of $\mathrm{C}^{n}$, J. Funct. Anal. 50 (1983), 100-126.

Department of Applied Mathematics, Korea Advanced Institute of Science and TechNology, P.O. Box 150, Cheong-Ryang, Seoul 130-650, Korea 